\title{
INTERPRETACIÓN SOCIAL Y MULTICULTURALIDAD: EL CASO DE LAS LENGUAS INDÍGENAS DE MÉXICO
}

\section{COMMUNITY INTERPRETING AND MULTICULTURALISM: THE CASE OF THE MEXICAN INDIGENOUS LANGUAGES}

\author{
Antonio Reyes Pérez \\ INSTITUTO SUPERIOR DE INTÉRPRETES Y TRADUCTORES, \\ LABORATORIO DE TECNOLOGÍAs LINGÜÍ́STICAS \\ Ximena Iglesias Carrillo \\ INSTITUTO SUPERIOR DE INTÉRPRETES Y TRADUCTORES, \\ LABORATORIO DE TeCNOLOGías Lingüísticas
}

\section{RESUMEN}

La interpretación social se entiende como un proceso de mediación en el que la comunicación sucede en un plano social que implica la interacción de grupos culturalmente diferentes, tales como migrantes, refugiados o comunidades indígenas (Pöchhacker, 2004; Hale, 2007). En este marco, el objetivo de este trabajo es exponer el escenario general que supone la interpretación social en México dada la gran diversidad lingüística que caracteriza a este país. Así, se enfatiza la problemática que debe resolver el intérprete social en tanto que se enfrenta a diferentes lenguas y diferentes culturas que coexisten en una realidad aparentemente homogénea. Finalmente, se muestran los avances y resultados que diversas instituciones públicas y privadas han hecho en torno a este fenómeno.

Palabras ClaVe: mediación lingüística; mediación cultural; lenguas indígenas mexicanas; interpretación; identidad cultural 


\section{Abstract}

Community Interpreting is a mediation process in which communication occurs within a social framework that entails the interaction of culturally different subgroups such as migrants, refugees, or indigenous communities (Pöchhacker, 2004; Hale, 2007). In this context, this article aims to describe the general setting in which Community Interpreting unfolds in Mexico, given the high linguistic diversity of this country. We emphasize the challenges that community interpreters face when coping with different languages and cultures coexisting within an apparently homogenous reality. Finally, we describe the progress made and results obtained by public and private institutions in this regard.

KEYWORDS: linguistic mediation; cultural mediation; Mexican indigenous languages; interpreting; cultural identity 


\section{INTRODUCCIÓN}

En términos generales, la interpretación social se ha definido como un proceso de mediación en el que la comunicación se realiza en un contexto social, que se caracteriza por tener un grupo principal o dominante y uno o varios subgrupos secundarios o dominados (Pöchhacker, 2004; Hale, 2007). Considérense, por ejemplo, grupos minoritarios tales como migrantes o refugiados que se insertan en un contexto nuevo y totalmente diferente de aquel del cual provienen. Entendida así, la interpretación social se ha relacionado estrechamente con los flujos migratorios (Wadensjö, 1998). La comunidad hispana en Estados Unidos o la población africana en Europa son una muestra. En este escenario, lograr una comunicación exitosa implica que el intérprete sea capaz de mediar no solo en términos de diferencias lingüísticas sino, particularmente, en términos de diferencias culturales.

Dado este contexto migratorio, la interpretación social se entiende como un fenómeno mucho más complejo y con múltiples dimensiones, puesto que las barreras léxicas o terminológicas se supeditan a aspectos menos asibles tales como la idiosincrasia, las particularidades culturales o, incluso, la propia cosmovisión. No obstante la complejidad que implica este tipo de interpretación, ha habido un esfuerzo constante para sistematizar la investigación y su metodología sobre este fenómeno. Por ejemplo, en algunas investigaciones se ha abordado la problemática de la interpretación social delimitada a contextos comunicativos particulares: situaciones legales, acceso a la salud, acceso a los servicios sociales, entre otros ( $c f$. Hertog \& van der Veer, 2006; Carr, Roberts, Dufour \& Steyn, 1997), otras investigaciones se han dedicado a analizar el rol, la ética o la afinidad del intérprete para con un grupo específico, o bien la necesidad de capacitación para resolver situaciones en diferentes escenarios interpretativos ( $c f$. Prunč, 2012; Vargas, 2012).

A pesar de la complejidad de la interpretación social, el trabajo del intérprete está totalmente delimitado porque se enfrenta a las realidades de dos grupos claramente definidos y diferenciables. Sin embargo, hay escenarios atípicos en los que la necesidad de una interpretación social no obedece a situaciones motivadas por cuestiones migratorias. México es uno de tales escenarios: el español es la lengua nacional del país; sin embargo, hay más de 11 familias lingüísticas representadas por 68 lenguas indígenas con 364 variantes (Inali, 2008, 2009). La sola cantidad de lenguas supone un gran reto para la interpretación social; no 
obstante, no es el número de lenguas lo que plantea el escenario atípico. La problemática que enfrenta el intérprete social en México tiene que ver con que tales lenguas, muchas de ellas con variantes dialectales muy importantes, representan culturas totalmente diferentes que coexisten dentro de una realidad aparentemente homogénea; $i$. e. hay tantas realidades codificadas a través de la lengua, todas ellas tan diferentes y tan particulares, que lo único que comparten es el hecho de que todos sus hablantes cohabitan en el mismo país, no por razones migratorias, sino porque todos son mexicanos originarios.

El objetivo de este artículo, considerando lo anterior, es exponer el escenario general que supone la interpretación social en México, así como describir los retos que esta implica y los avances que tanto instituciones públicas como privadas han hecho al respecto.

El artículo está organizado de la siguiente forma. En §2 se describen algunos de los trabajos más importantes en el marco de la interpretación social, enfatizando aquellos que pueden vincularse con el escenario mexicano. En $\S 3$ se detallan las características generales de la situación sociocultural y lingüística que priva en México. En $§ 4$ se expone la situación actual y las prospectivas de la interpretación social en México. Por último, en $\$ 5$ se aportan las consideraciones finales y se plantean algunas líneas de trabajo futuro.

\section{ESTADO DEL ARTE: TRABAJOS SOBRE INTERPRETACIÓN SOCIAL}

La atención que se ha puesto en las últimas décadas en los derechos humanos, así como el aumento de las olas migratorias, han aumentado la conciencia sobre la necesidad del servicio de interpretación en el sector social; por tanto, muchos gobiernos, asociaciones y grupos de investigación están desarrollando estudios y proyectos en este ámbito.

Pöchhacker (2004) define la interpretación social como un contacto intrasocial, que se desarrolla en contextos multiculturales y en escenarios tales como tribunales, comisarías, hospitales o dependencias de la administración pública principalmente, pues al fin y al cabo el intérprete social siempre trabaja en ámbitos institucionales.

Cada país ha desarrollado sistemas propios para proveer servicios de interpretación social: desde la capacitación y certificación de los intérpretes hasta la puesta en marcha de una estructura nacional para brindar servicios oportunos y 
de calidad. En ese sentido, Australia, Canadá, España, Estados Unidos, Francia, Reino Unido y Suecia son ejemplos claros de los avances que pueden lograrse desde instituciones gubernamentales, no gubernamentales y académicas.

Así, en cuanto a los contextos de actuación, dentro de un marco jurídico (tribunales, comisarías, audiencias) destacan los trabajos de Mikkelson (1999), Pöllabauer (2004) y Hale (2007). En el ámbito médico, destacan las investigaciones de Hale (2007), mientras que, en cuanto a los servicios públicos y la terminología, los de Valero-Garcés $(2005,2008)$ son referente.

Ahora bien, debido a los contextos sensibles, e incluso de vulnerabilidad, en los que se desarrollan los servicios de interpretación social, la labor del intérprete debe estar regida por códigos deontológicos que orienten su labor. En este sentido, los trabajos de Angelleli (2004) y Hale (2007) abordan el rol que juega el intérprete desde tal ángulo.

Finalmente, las propuestas de Abril (2006), Hale (2007) y Valero-Garcés (2008) son referencias valiosas en cuanto al proceso de formación del intérprete.

\section{Situación Sociocultural y Lingüística en MÉXico}

Ninguna sociedad en el mundo es uniforme en su composición. México no es la excepción, pues se reconoce y se identifica como un país único e indivisible, pero culturalmente plural. Así, su Constitución política reconoce esta pluriculturalidad en el caudal de pueblos originarios, con más de 11 millones de indígenas que habitan en el territorio nacional, lo que representa $10 \%$ del total de la población (Inali, 2009), situación que convierte a México en el país que alberga el mayor número de indígenas en el continente americano (Zolla \& Zolla Márquez, 2010: 41).

\subsection{Marco sociocultural}

A partir de los censos de población y vivienda, así como de lo plasmado en el Catálogo de las Lenguas Indígenas Nacionales: variantes lingüísticas de México con sus autodenominaciones y referencias geoestadísticas (Inali, 2008), se sabe que México cuenta con 11 familias lingüísticas, de las que se desprenden 68 agrupaciones con 364 variantes. No obstante esta riqueza lingüística, el hecho de que coexistan junto a una lengua con mayor peso demográfico como lo 
es la española, causa que se privilegie el uso de esta al funcionar como punto de enlace. Esta situación, que tiene su origen en diferencias culturales y, obviamente, lingüísticas, ha sido una barrera que históricamente ha impedido que la población indígena tenga total reconocimiento social, así como acceso pleno a los servicios públicos a los que por ley tiene derecho.

El escaso respeto y valoración de la riqueza cultural y lingüística de lo indígena se remonta a la época del Virreinato, en la que el "indio" era visto como un ser de naturaleza inferior, lo que resultó en su segregación social, política y económica, así como en su exclusión de los servicios públicos, entre los que se cuentan la educación y la salud. Hasta principios del siglo xx, en México, ser indígena (en el sentido más puro: persona originaria de un país) era motivo de discriminación y sello de vergüenza, lo que conllevó a la negación de las más mínimas posibilidades de incorporación a la vida nacional (Inali, 2009). Luego de 1920, con la Revolución mexicana se fundamentó la idea de que la unidad nacional y la igualdad de derechos equivalían a homogeneizar culturalmente al país, pues la diversidad era vista como un obstáculo para caminar hacia la modernidad. Hasta hace apenas 20 años seguía privando la falta de respeto a lo diferente y el discurso oficial insistía en que sería el monoculturalismo el que resarciría la deuda histórica que se tenía con los indígenas, pues solo se podían superar los rezagos sociales si los indígenas anulaban su pasado, su identidad y su lengua para adoptar la cultura mexicana (Inali, 2009). Así, el Estado desplegó un conjunto de políticas para promover la asimilación de los indígenas a la cultura nacional y tratar lo indígena como pieza de museo. En este sentido, el desconocimiento oficial de lo que en verdad comporta el ser indígena devino en la formulación de políticas desarticuladas, a pesar de que, para ese entonces, México ya había ratificado numerosos tratados, leyes y convenciones internacionales.

No es sino hasta el año 2003, con la promulgación de la Ley de la Comisión Nacional para el Desarrollo de los Pueblos Indígenas y la Ley General de Derechos Lingüísticos de los Pueblos Indígenas, cuando las políticas sociales plantean una visión distinta en favor de la pluralidad cultural, y en su propia instrumentación se reconoce el uso de las lenguas indígenas. Así, uno de los principales logros de estas leyes ha sido declarar las lenguas indígenas y el español como lenguas nacionales, además de reconocer el derecho de los mexicanos a hablar su lengua materna, sin ningún tipo de limitación, en cualquier ámbito. 


\subsection{Marco lingüístico}

La lengua es un elemento cultural único que representa la esencia de un pueblo, en tanto que lo define, le da identidad y le hace ser lo que es; cada lengua, por tanto, manifiesta una cosmovisión que constituye una manera de ver el mundo y que da sentido de pertenencia a una comunidad (CDI, 2010: 17, 21). De acuerdo con datos presentados por el Instituto Nacional de Lenguas Indígenas (Inali, 2008, 2009), México es una nación plurilingüe en la que, además del español, coexisten 68 lenguas con más de 360 variantes que se agrupan en 11 familias lingüísticas (véase Cuadro 1).

Cuadro 1. Familias lingüísticas en México

\begin{tabular}{lll}
\hline álgica & yuto-nahua & cochimí-yumana \\
seri & oto-mangue & maya \\
totonaco-tepehua & tarasca & mixe-zoque \\
chontal de Oaxaca & huave & \\
\hline
\end{tabular}

El escenario que plantea esta diversidad de lenguas en contacto, que en muchos casos están supeditadas a la lengua mayoritaria, hace que un trabajo en interpretación social sea imperativo; sobre todo tomando en cuenta que a) muchos de esos hablantes solo hablan una lengua indígena y b) esos mismos hablantes tienen necesidades que no están motivadas por las mismas causas que, por ejemplo, supondría una interpretación social en un marco de flujos migratorios.

Cabe destacar, además, que el hecho de que existan distintas variantes de estas lenguas implica que no haya una inteligibilidad total entre los hablantes. Por tanto, los procesos comunicativos que debe enfrentar el intérprete se vuelven mucho más complejos. A continuación se detallan algunos aspectos de las lenguas indígenas que coexisten en México con la finalidad de contextualizar la situación que enfrenta el intérprete social en un escenario como el mexicano.

\subsection{Lenguas indígenas}

De las 68 lenguas anteriormente referidas, las más representativas en términos del número de hablantes son el náhuatl, el maya, el mixteco y el zapoteco. La 
primera con más de un millón de hablantes, la segunda con alrededor de 800000 hablantes, en tanto que las dos últimas con poco más de 400000 hablantes cada una (cf. Inali, 2009). Del resto de las lenguas, algunas no llegan a los 1000 hablantes, mientras que algunas otras están en vías de extinguirse. En la Figura 1 se puede observar la distribución de las 11 familias lingüísticas en términos del número de hablantes.

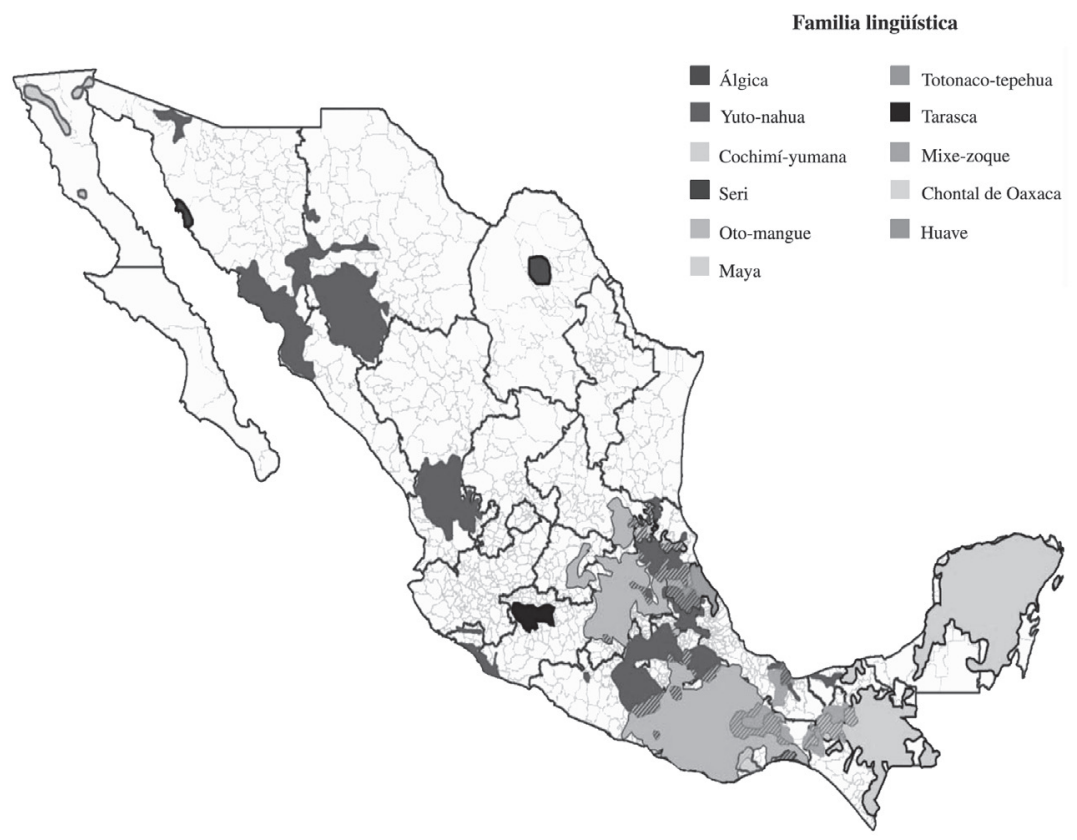

Figura 1. Distribución de las familias lingüísticas indoamericanas en México. Mapa adaptado de Inali (2009: 16)

La distribución territorial es un punto relevante que influye directamente en la problemática que enfrenta el intérprete social en México (en \$3.2. se aborda de manera detallada dicha problemática). Como se observa en la Figura 1, la mayoría de las lenguas se concentra en el centro del país y hacia el sur. Esto es sumamente importante porque tanto aspectos geográficos, climáticos como económicos y fenotípicos son diametralmente diferentes en el norte, centro y sur del país. Así, las necesidades que puede tener un hablante seri del desierto de Sono- 
ra (norte de México) no serán las mismas que las de un hablante chontal de la selva de Chiapas (sur de México).

Asimismo, otro punto importante que impacta a estas lenguas tiene que ver con su estudio y documentación. Si bien lenguas como el maya o el náhuatl han sido bastante estudiadas y descritas, incluso en términos de variantes dialectales, hay lenguas que no lo han sido. La consecuencia inmediata, al menos para el trabajo del intérprete, es una falta de materiales que le permitan, primero, conocer la estructura de la lengua y, después, sistematizar su conocimiento a partir de una norma consensuada que facilite su trabajo de mediación.

\subsection{Identidad cultural}

La diversidad lingüística arriba descrita convierte al país en una nación pluricultural. La lengua hace que las personas que comparten un mismo código se reconozcan como pertenecientes a un núcleo común, en tanto que las diferencia de quienes no lo comparten. En el contexto nacional, no obstante que quienes nacieron en el país comparten la noción de ser "mexicanos", la lengua que hablan los diversos grupos humanos hace que entre ellos se perciban con más diferencias que similitudes. En particular si, durante siglos, se ha vivido con el estigma de que hablar una lengua que no es la "oficial" (i. e. una lengua indígena) significa vivir en desventaja; significa no ser igual.

Ahora bien, las diferencias lingüísticas implican una identidad cultural claramente definida. Es a través de la lengua que filtramos nuestras percepciones del mundo, nuestra cosmovisión. En este sentido, es importante señalar que el hecho de reconocer la existencia de varias familias lingüísticas, con sus múltiples lenguas y variantes, significa aceptar que "la población originaria de lo que actualmente es el territorio nacional [México] no compartió en la antigüedad la misma historia sociocultural, sino que se desarrolló sobre diferentes experiencias en tiempos y espacios presumiblemente disímiles también" (Inali, 2008: 38).

Para el intérprete social en México, lo anterior supone el escenario atípico del que se habló en secciones previas: además de mediar entre lenguas, debe hacerlo entre culturas que, a pesar de que se suscriben a un mismo concepto de nación, tienen una cosmovisión y, en consecuencia, un conjunto de necesidades radicalmente diferentes, mismas que probablemente sean incomprensibles para quienes son ajenos a ellas. Por ejemplo, la realidad de un indígena del sur de 
México es, desde todos los ángulos, distinta a la de un indígena del norte, y no hablamos específicamente de diferencias obvias, sino de diferencias que se sustentan en la manera en la que se conforma una cultura determinada. Incluso, la realidad para los hablantes de dos variantes dialectales no es, de ningún modo, igual. Así, un hablante de náhuatl de los suburbios de la Ciudad de México tendrá necesidades, motivaciones y, posiblemente, una cosmovisión diferente a la de un hablante de la variante hablada (y conceptualizada) de algún municipio de la sierra de Veracruz. Así, mientras que para uno la naturaleza será un elemento relevante que el intérprete debería considerar, para otro lo será el elemento ritual.

\section{INTERPRETACIÓN SOCIAL EN MÉXICO}

Como ya se ha expuesto, en México no solo cohabitan varias lenguas indígenas, sino que cada lengua en sí misma representa una cultura distinta. A pesar de que las manifestaciones humanas son diversas desde su origen, los ordenamientos internacionales y nacionales en cuanto al reconocimiento de los pueblos indígenas y sus expresiones culturales, como la lengua, son relativamente recientes, según se describe a continuación.

\subsection{Contexto legislativo}

En el contexto mexicano, la proclamación de ordenamientos en materia de derechos de los pueblos indígenas y minorías lingüísticas es reciente. Al margen de las ratificaciones hechas por México a las leyes y los pactos internacionales relacionados con estos temas, la primera acción del Estado mexicano no fue sino hasta 1992, con la reforma del artículo 4 constitucional, en el que se reconoce por primera vez a los pueblos indígenas y se obliga a respetar sus culturas (Inali, 2009) y la reforma del artículo 102, en el que se estipula la protección y la defensa de los derechos humanos a partir de la creación de la Comisión Nacional de los Derechos Humanos.

En 2003, con la promulgación de la Ley de la Comisión Nacional para el Desarrollo de los Pueblos Indígenas se planteó por primera vez la participación del Estado en actividades de apoyo, fomento y coordinación de programas y acciones públicas para el desarrollo de los pueblos indígenas. 
A su vez, esta ley promovió la creación de la Ley General de Derechos Lingüísticos de los Pueblos Indígenas y la consecuente fundación del Instituto Nacional de Lenguas Indígenas. Desde su concepción, el Inali se dedicó, en primera instancia, a clasificar las lenguas indígenas nacionales para luego dirigir sus esfuerzos, junto con otras instancias tanto públicas como privadas, a delinear procesos que ayudaran a la conformación de una estructura cohesionada en cuanto a las lenguas indígenas y los procesos de mediación lingüística y cultural se refiere. Cabe mencionar que los hablantes de lenguas indígenas tienen garantizado el derecho a ser asistidos por intérpretes, traductores y defensores públicos profesionales, con un conocimiento amplio y profundo de la lengua y cultura; de este modo, los indígenas son tratados con base en sus usos y costumbres.

\subsection{Intérpretes}

En la actualidad, en México existen dos tipos de intérpretes de lenguas indígenas: los formados a través de la práctica de saberes culturales, lingüísticos y propios de sus pueblos originarios y los formados a través de procesos académicos formales y de evaluación de competencias laborales para la interpretación.

Ambos tipos de intérpretes pueden acreditarse y/o certificarse mediante los mecanismos que a continuación se exponen, los cuales tienen como fin generar condiciones de accesibilidad y equidad en el trato para los hablantes de lenguas indígenas.

\subsubsection{Capacitación y certificación}

A pesar de que en México existen programas de formación profesional en interpretación desde hace décadas, no fue sino hasta 2007 que se instauró el primer programa de capacitación para intérpretes en lenguas indígenas, con el propósito de que existan profesionales certificados en todas las lenguas y para los diferentes tipos de servicios públicos en que sean necesarios.

El primer diplomado de este tipo se realizó en la Ciudad de México, y en los años subsiguientes se han impartido en más de 10 de los 31 estados de la república; no obstante, solo se enfocan en los ámbitos de procuración, impartición y administración de justicia. 
Los diplomados son gratuitos y tienen una duración de 180 horas. En estos se tratan temas relacionados con teoría y práctica de la interpretación, aspectos lingüísticos, aspectos jurídicos, derechos de los pueblos y comunidades indígenas, así como temas afines a las lenguas y las culturas indígenas de la región en la que se desarrolla el curso. Los aspirantes deben ser mayores de edad, que en el caso que nos ocupa es 18 años; ser hablantes de alguna variante de las lenguas oficiales; saber hablar, leer y escribir en español; contar con actitudes positivas hacia su lengua y su cultura originaria, y tener habilidades para la interpretación.

Una de las grandes dificultades de estos programas de capacitación es que los participantes han de recorrer grandes distancias en lugares que no cuentan con una infraestructura adecuada en el plano de las comunicaciones.

En 2008 se formó, junto con el Inali, el Comité de Normalización de Asuntos Indígenas para crear el Estándar de Competencia Laboral para la Función de Interpretación Oral de Lengua Indígena al Español y Viceversa en el Ámbito de Procuración y Administración de Justicia, que se publicó en el Diario Oficial de la Federación en 2009. Esta norma es referente para la evaluación y certificación de las personas que se desempeñan como intérpretes en procesos de procuración y administración de justicia en los que esté involucrado una persona hablante de lengua indígena y se requiera entablar con ella una comunicación oral efectiva.

Por otra parte, con el fin de reconocer la experiencia de intérpretes prácticos que realizan labores de interpretación, así como de fortalecer los procesos de formación en los que los intérpretes indígenas formen a otros indígenas, a partir de 2011 el Inali está desarrollando un Diplomado Piloto para la Formación de Formadores de Intérpretes de Lenguas Indígenas, impartido por egresados de procesos tanto de formación y acreditación de intérpretes como de certificación de competencias laborales.

\subsubsection{Ejercicio profesional}

A pesar de la imperiosa necesidad que existe de intérpretes en los servicios públicos y de los esfuerzos realizados por numerosas instituciones, el número de intérpretes que hay en México todavía es escaso, y no todos han recibido la capacitación adecuada.

El Padrón Nacional de Intérpretes y Traductores en Lenguas Indígenas (Panitli), creado por el Inali, registra apenas 575 intérpretes que han sido acredita- 
dos y/o certificados, aunque en la práctica son muchos más los que ejercen activamente la disciplina, a pesar de no estar debidamente reconocidos.

Ahora, como se expuso anteriormente, uno de los problemas principales que impera en México en cuanto a la interpretación en lenguas indígenas es que hay tantas variantes dialectales que, aunque están recogidas en el Catálogo de las Lenguas Indígenas Nacionales, resulta difícil determinar oralmente si dos hablantes hablan la misma variante hasta que no se comunican entre ellos y comprueban que en su comunicación existe inteligibilidad. Por ello, a últimas fechas se ha optado por preguntar el lugar de origen del indígena que necesita los servicios de interpretación para saber con mayor certeza la variante dialectal de la que se trata.

En cuanto a la jornada laboral de un intérprete de lenguas indígenas, aún no existe legislación alguna sobre el número de horas que conforman una jornada, ni sobre si ha de trabajar en conjunto con otros intérpretes. A pesar de que la ley señala que los intérpretes podrán cobrar honorarios por su trabajo, en la práctica esto no ha sido así, pues todavía se presentan casos en los que no reciben pago alguno. En numerosas ocasiones, las mismas instancias gubernamentales carecen de recursos para cubrir estos gastos.

Al igual que casi todas las profesiones, los intérpretes de lenguas indígenas cuentan con un marco de principios y valores que guían el comportamiento que han de observar en su ejercicio profesional. Fue el Inali el organismo que propuso un código de ética específico para estos intérpretes, que considera valores como la honestidad, la libertad, la justicia, la verdad y principios como el respeto, la no discriminación y la imparcialidad.

Finalmente, los intérpretes en lenguas indígenas se han agrupado libremente en dos esfuerzos concretos para emprender proyectos que beneficien a los propios intérpretes, así como a los indígenas que necesiten de sus servicios: la Organización de Traductores, Intérpretes Interculturales y Gestores en Lenguas Indígenas (OTIGLI) y la Organización Mexicana de Intérpretes Traductores de Lenguas Indígenas. 


\subsection{Retos}

Pese a las acciones aquí descritas para desarrollar un marco formal a nivel nacional para el correcto ejercicio de la interpretación social en lenguas indígenas, la actividad es sumamente reciente y poco reconocida, por lo que queda mucho por hacer.

A pesar de que el Catálogo de Lenguas Indígenas Nacionales (Inali, 2008) es la obra de referencia en su área, la identificación de las variantes dialectales en la práctica cotidiana sigue siendo un escollo. Además, la distancia geográfica entre comunidades, así como los deficientes sistemas de comunicaciones y transportes causan que la prestación de los servicios de interpretación sea complicada de lograr y, por tanto, sea considerada como un proceso en desarrollo.

En lo concerniente a los procesos de formación, no se han desarrollado programas que impartan conocimientos sobre mediación lingüística en otros ámbitos más allá del de procuración, impartición y administración de justicia. Cabe añadir que, si bien las horas que se imparten (180) es un número importante, hay que considerar que ese tiempo es insuficiente para tratar la complejidad que supone formar a intérpretes en lenguas indígenas, más aún si se toma en cuenta que la selección de candidatos no cuenta con parámetros objetivamente verificables fuera de los aquí descritos.

En ese sentido, junto con los programas de formación han de promoverse esfuerzos de compilación, sistematización y unificación de terminología en aquellos ámbitos de conocimiento donde existe mayor necesidad de servicios de interpretación. Asimismo, en el nuevo sistema de justicia penal establecido en México, ${ }^{1}$ el papel del intérprete tiene una relevancia fundamental dentro de los procesos; no obstante, aún no se ha delineado de manera puntual lo relacionado en cuanto a su actuación específica.

Por último, no obstante el esfuerzo de algunas instituciones por constituir un escenario estable y propicio para el desarrollo de la interpretación social en México, el número de instituciones abocadas a ello es aún escaso: el Inali, el Centro

1 A partir del año 2008 hubo una reforma constitucional al sistema jurídico mexicano en la que se busca mejorar la impartición de justicia a través de la realización de juicios orales. Es importante destacar que dicha reforma tiene un impacto directo en tanto la necesidad de contar con intérpretes que puedan asistir los procedimientos de mediación lingüística, cuando sea el caso, que garanticen la correcta procuración de justicia. 
Profesional Indígena de Asesoría, Defensa y Traducción, la Comisión Nacional para el Desarrollo de los Pueblos Indígenas y el Instituto Superior de Intérpretes y Traductores. El resultado, en consecuencia, es que dicho esfuerzo pareciera ser insuficiente dada la necesidad que plantea la riqueza lingüística mexicana.

\section{CONSIDERACIONES FINALES Y TRABAJO FUTURO}

En este trabajo hemos descrito, de manera general, el escenario que el intérprete social enfrenta en México. Es importante resaltar que si bien ha habido un esfuerzo sumamente importante en este ámbito en distintos países, en el caso de México, su contexto particular dificulta que no se puedan copiar, o incluso adaptar, los modelos que han tenido éxito en otros lugares. El número de lenguas y variantes dialectales, así como la diversidad cultural que conlleva cada una de ellas, hacen que la interpretación social en México sea un trabajo en constante proceso y lleno de retos.

Para concluir, se plantea como línea inmediata de trabajo el estudio linguiístico, terminológico, social y cultural de las lenguas indígenas que, como el maya, tienen diversas variantes dialectales con el fin de analizar, a partir de datos reales, la problemática, las necesidades y las posibles líneas de acción que a la postre permitan desarrollar modelos de interpretación social acordes con la realidad lingüística mexicana.

\section{REFERENCIAS}

Abril Martí, María IsABel (2006). La interpretación social en los servicios públicos: caracterización como género, contextualización y modelos de formación. Hacia unas bases de diseño curricular (Tesis doctoral inédita). Universidad de Granada, España.

Angelleli, Claudia V. (2004). Revisiting the interpreter's role. A study of conference, court, and medical interpreters in Canada, Mexico and United States. Ámsterdam: John Benjamins.

Carr, Silvana E.; Roberts, Roda P.; Dufour, Aideen, \& Steyn, Dini (Eds.) (1997). The critical link: Interpreters in the community. Papers from the 1st International Conference on Interpreting in Legal, Health and Social Service Settings (pp. 211-234). Ámsterdam: John Benjamins.

Comisión Nacional para el Desarrollo de los Pueblos Indígenas, CDi (2010). Programa para el Desarrollo de los Pueblos Indígenas 2009-2012. México: CDI.

Hale, Sandra Beatriz (2007). Community Interpreting. Londres: Palgrave McMillan. 
Hertog, Erik, \& van der Veer, Bart (2006). Taking stock: research and methodology in Community Interpreting. Linguistica Antverpiensia, 5, 11-17.

Instituto Nacional de Lenguas Indígenas, Inali (2008). Catálogo de las Lenguas Indígenas Nacionales: variantes lingüísticas de México con sus autodenominaciones y referencias geoestadísticas. Diario Oficial de la Federación, 14 de enero de 2008.

Instituto Nacional de Lenguas Indígenas, Inali (2009). Programa de revitalización, fortalecimiento y desarrollo de las lenguas indígenas nacionales 2008-2012, Pinali. México: Instituto Nacional de Lenguas Indígenas.

Mikkelson, Holly (1999). Court interpreting at a crossroads. Annual Conference of the National Association of Judiciary Interpreters and Translators (NAJIT), May 1999. Monterey Institute of International Studies. Recuperado de https://works.bepress.com/holly_mikkelson/10/

PöchHacker, Franz (2004). Introducing interpreting studies. Londres: Routledge.

Pöllabauer, Sonja (2004). Interpreting in asylum hearings. Issues of role, responsibility and power. Interpreting, 6(2), 143-80.

PRUNČ, ERICH (2012). Rights, realities and responsibilities in Community Interpreting. The Interpreters' Newsletter, 17, 1-12.

Valero-Garcés, Carmen (2005). Terminology and ad hoc interpreters in public services. An empirical study. Jostrans: The Journal of Specialised Translation, 3, 75-96.

Valero-Garcés, Carmen (2008). Hospital interpreting practice in the classroom and the workplace. En Carmen Valero-Garcés \& Anne Martin (Eds.). Crossing borders in Community Interpreting. Definitions and dilemmas (pp.166-85). Universidad de Alcalá: John Benjamins.

VARgas UrPI, Mireia (2012). State of the art in Community Interpreting research. Mapping the main research topics. Babel, 58(1), 50-72.

WADENSJÖ, CECILIA (1998). Interpreting as interaction. Londres: Longman.

Zolla, Carlos, \& Zolla Márquez, Emiliano (2010). Los pueblos indígenas de México: 100 preguntas. México: Universidad Nacional Autónoma de México. 